\title{
Femmes et entrepreneurs : Trente ans de recherches en motivation entrepreneuriale féminine
}

\author{
Aude d'Andria et Inés Gabarret
}

\section{Résumé}

L'entrepreneuriat féminin a été largement étudié par rapport à celui des hommes. Pouvons-nous, néanmoins, encore parler d'un entrepreneuriat féminin unique ? Le panorama de l'entrepreneuriat féminin s'étant complexifié, différents profils motivationnels peuvent coexister. Dans cet article, à partir d'une revue approfondie de la littérature, nous parcourons trente ans de recherches en motivation entrepreneuriale féminine. Notre étude fait ressortir trois idéaux-types qui apparaissent de manière régulière dans la littérature: les femmes qui entreprennent par nécessité, les entrepreneures (en transition) de carrière et les femmes qui entreprennent au moment de la maternité (mampreneurs). Nous concluons que la recherche en entrepreneuriat féminin doit tenir compte de l'hétérogénéité des femmes, et que leurs motivations ne peuvent être étudiées en dehors de la relation au cycle de vie de la femme et de la famille.

Mots clés : entrepreneuriat féminin, entrepreneure, mampreneur, motivation entrepreneuriale, nécessité/opportunité, push/pull.

\section{Introduction}

Caractérisée comme adolescente par Hughes et al. (2012), la recherche en entrepreneuriat féminin a vu ses premiers articles il y a une trentaine d'années (Jennings et Brush, 2013). En dépit de leur développement et de leur contribution économique significative partout dans le monde (OCDE, 2012; GEM, 2015), la thématique des femmes qui entreprennent reste encore sous-étudiée (Brush et Cooper, 2012). Elle a cependant atteint «une maturité théorique incontestable et fait partie, à part entière, de la recherche académique sur l'entrepreneuriat » (Léger-Jarniou, Nelson et Chasserio, 2015 : 19). Comme le rappellent ces auteures, des préjugés conscients ou inconscients, mais bien enracinés, demeurent chez l'ensemble des acteurs, y compris chez les chercheurs et les entrepreneur(e)s ${ }^{1}$.

Comme l'avait déjà exprimé Neider (1987) il y a presque 30 ans, à partir des résultats de sa recherche menée sur un groupe d'entrepreneures, nous faisons nôtre l'idée d'une hétérogénéité en entrepreneuriat féminin. En effet, Neider montrait que les femmes âgées de plus de 50 ans, correspondaient à une première génération des femmes qui avaient créé suite à des crises personnelles (divorce, mort de l'époux, etc.) et celles âgées de moins de 50 ans l'avaient fait afin de poursuivre un désir de satisfaction

\footnotetext{
${ }^{1}$ Voir le numéro spécial de la Revue de l'Entrepreneuriat, vol.14, $n^{\circ} 2-3$ consacré aux femmes entrepreneurs
} 
personnelle. Elle expliquait ces résultats comme l'avènement d'une seconde génération d'entrepreneures.

Presque vingt années plus tard, Ahl (2006) proposait de donner une plus grande attention à l'étude de l'hétérogénéité des femmes et aux différents contextes dans lesquels ces femmes sont intégrées. La même année, au regard de l'accroissement de la création d'entreprises par les femmes au Canada, Hughes (2006) montrait la diversité de profils d'entrepreneures canadiennes, tant en ce qui concerne leurs motivations, que leur performance et la réussite de leurs entreprises. Elle identifiait trois types de motivations entrepreneuriales des femmes à savoir : classique, forcée, et travail-famille. Depuis, le champ d'étude de l'entrepreneuriat féminin s'est considérablement étendu, avec le développement de conférences, de revues spécialisées, de rapports en entrepreneuriat féminin $^{2}$, et de livres qui traitent spécifiquement de l'entrepreneuriat des femmes (Hughes et al., 2012). Ces travaux apportent des contributions significatives dans la recherche en entrepreneuriat, principalement sur quatre aspects : (1) l'entrepreneuriat comme un phénomène genré, (2) encastré dans une réalité de famille, (3) résultant aussi bien d'une nécessité que d'une opportunité, et (4) poursuivant des objectifs qui ne sont pas seulement économiques (Jennings et Brush, 2013).

Dans cet article, nous nous intéressons à l'évolution de la recherche en motivation entrepreneuriale féminine. La motivation entrepreneuriale, qui est aussi à la base des typologies d'entrepreneur, reste un sujet d'actualité (Carsrud et Brannback, 2011). Que pouvons-nous retenir de ces trente années de recherche sur la motivation entrepreneuriale des femmes ? Le panorama de l'entrepreneuriat féminin s'étant complexifié, nous nous demanderons quels sont les principaux profils d'entrepreneures étudiés dans la littérature?

Notre article commence par une présentation du contexte des études en entrepreneuriat féminin et de la méthode de sélection de notre revue de littérature. Les principales recherches en motivation entrepreneuriale féminine et les typologies basées sur des facteurs de motivation sont ensuite discutées. Trois idéaux-types ${ }^{3}$ sont identifiés. Nous concluons que la recherche en entrepreneuriat féminin doit tenir compte de l'hétérogénéité des femmes, et que leurs motivations ne peuvent être étudiées en dehors de la relation au cycle de vie de la femme et de la famille (Aldrich et Cliff, 2003 ; Brush et Cooper, 2012).

\section{Le contexte de la recherche}

Si la plupart des recherches ont entretenu, malgré elles, des mythes et des stéréotypes, c'est parce qu'elles ont longtemps eu tendance à considérer l'entrepreneuriat en général, et l'entrepreneuriat féminin en particulier, comme une réalité homogène. Ainsi, au-delà

\footnotetext{
${ }^{2}$ Comme par exemple le rapport 2015 sur l'entrepreneuriat féminin du Global Entrepreneurship Monitor

${ }^{3}$ Un idéal-type au sens de Weber (1904-1917) est une catégorie «abstraite » de l'existant, qui permet de proposer une synthèse, en accentuant certaines caractéristiques jugées représentatives d'un fait ou d'un ensemble de faits ayant cours dans l'histoire. Elle aide à comprendre ou à théoriser certains phénomènes, sans prétendre que les caractéristiques de l'idéal-type se retrouvent toujours dans les phénomènes observés.
} 
des huit mythes repérés par Brush et al. $(2001)^{4}$ sur les femmes qui entreprennent, l'un des tout premier a été d'associer implicitement l'entrepreneuriat au genre masculin (Hisrich et Brush, 1984 ; Stevenson, 1990 ; de Bruin et al., 2006, 2007 ; Hill et al., 2006 ; Gawel, 2013 ; Lewis, 2013; Verduijn et Essers, 2013). Dès lors, l'entrepreneuriat étant perçu originellement comme une question d'hommes, la discussion s'est développée en rendant compte de la similitude ou de la différence entre les hommes et les femmes (Ferraz Gomes et al., 2014). Les premières recherches des années 1970 ont plutôt été marquées par l'étude de la différence entre les sexes (biologiques), puis celles menées dans les années 1990, ont plutôt considéré l'entrepreneuriat comme une activité ne variant pas d'après le genre (Birley, 1989 ; Buttner et Moore, 1997, Holmquist, 1997). Les années 2000 ont ensuite vu le retour de l'étude de la différence entre les hommes et les femmes (Kirkwood, 2009; Gupta et al., 2009 ; Brush et Cooper, 2012 ; Gawel, 2013 ; Jennings et Brush, 2013). Les femmes chefs d'entreprise sont alors désignées par le mot «femme » placé à coté de celui d'entrepreneur. Cela signale leur différence par rapport à la norme «masculine », en mesurant leur performance et leur comportement en tant qu'entrepreneurs (Lewis, 2006).

Les recherches tendent aujourd'hui à montrer qu'il y a plus de similitudes que de différences entre hommes et femmes entrepreneurs (Brush, 1992 ; Neergaard et al., 2005 ; Ahl, 2006 ; Pines et al, 2010). Par conséquent, le questionnement n'est plus de savoir si le genre est source de différence, mais de comprendre comment (et en quoi) il fait la différence (Pines, Lerner et Schwartz, 2010).

Dans ce contexte, il importe de reconnaître que ni les entrepreneures, ni leurs entreprises ne sont homogènes parce que leurs vécus, leurs aspirations, leurs expériences, les contextes dans lesquelles elles entreprennent... sont différents (Carter, 2000 ; Gundry et al., 2002 ; Marlow et Carter, 2004 ; Hill et al., 2006). En outre, leurs motivations et les types d'entreprise qu'elles vont créer ne sont pas indépendants de leur situation de famille. Les choix des femmes sont liés au cycle de vie de la famille, et les opportunités perçues ou découvertes, les financements disponibles et les processus développés seront différents selon les moments de la vie et face à divers contextes. Cela renvoie au concept d'encastrement ou d'enchâssement (« embeddedness » en anglais) qui est présent entre l'entrepreneuriat et la famille. Par conséquent, non seulement le système familial va affecter la création d'entreprise mais la réciproque est également vraie (Aldrich et Cliff, 2003).

\section{La méthode de recherche}

Notre revue de littérature s'est construite en deux temps. Tout d'abord, nous nous sommes appuyés sur la base des articles analysés par Jennings et Brush (2013) issus de

\footnotetext{
${ }^{4}$ Les huit mythes repérés par Brush et al. (2001) sont que les femmes : (1) ne veulent pas posséder des entreprises à forte croissance ; (2) n'ont pas les bons référents éducatifs antérieurs ni (3) les bons types d'expériences nécessaires pour construire de grandes entreprises ; (4) n'ont pas les bons réseaux et liens sociaux pour construire des entreprises crédibles; (5) ne disposent pas de ressources financières suffisantes pour lancer des entreprises à forte croissance ; (6) ne présentent pas de business plan, (7) se concentrent dans les secteurs non attractifs et (8) ne représentent pas une force pour les capital-risqueurs.
} 
leur étude sur la thématique de l'entrepreneuriat féminin et publiée dans Academy of Management Annals. Au total, les auteurs retiennent 630 articles parus entre 1975 et 2012 dans la littérature anglo-saxonne.

La recherche a été effectuée dans EbscoHost (Business Source Premier) en fonction des possibilités matérielles d'accès aux articles. Les auteurs ont sélectionné les articles à partir des résumés et n'ont retenu que ceux qui se focalisaient sur la thématique de la motivation entrepreneuriale des femmes. Des mots clés ont été utilisés, comme par exemple : «entrepreneurial motivations », «push and pull factors », «necessity and opportunity», «feminin or women entrepreneurship » ainsi que ceux qui s'occupaient des différences entre les femmes qui entreprennent. Plus précisément, les publications sélectionnées répondaient à deux critères :

- l'explicitation des facteurs de motivations entrepreneuriales des femmes ;

- les typologies d'entrepreneurs au féminin.

Pour les années ultérieures à 2013 (allant de 2013 à 2015), nous avons ensuite suivi la liste des revues proposée par Fayolle (2012) qui retient 7 revues dédiées à l'entrepreneuriat et 4 revues généralistes ${ }^{5}$. Nous avons ajouté à cette liste la revue International Journal of Gender and Entrepreneurship (IJGE), première revue académique de recherches sur cette thématique apparue en 2009, et Gender, Work and Organization $(G W O)$.

Cette liste ne prenant pas en compte la recherche francophone, nous avons également intégré dans notre sélection les articles issus de deux revues francophones en entrepreneuriat: la Revue de l'Entrepreneuriat et la Revue Internationale PME, ainsi que deux revues qualifiées de généralistes : La Revue française de gestion (RFG) et La Revues des Sciences de Gestion ${ }^{6}$. La recherche a été effectuée cette fois dans Cairn.

Nos recherches couvrent une période de trente ans. Elles commencent par des articles tels celui de Scott (1986), dans lequel l'auteure cherche à identifier les raisons de l'augmentation du nombre d'entrepreneures aux Etats-Unis, ainsi que celui de Bowen et Hisrich (1986), sur le modèle et les déterminants de la carrière entrepreneuriale des femmes. Notre recueil de littérature tient également compte des articles parus dans le récent numéro spécial consacré aux femmes qui entreprennent publié dans cette même revue sous la direction de Léger-Jarniou, Nelson et Chasserio (2015).

Au total, 82 articles et rapports ont été retenus pour nourrir notre revue de littérature sur les motivations des entrepreneures et sur leurs caractéristiques (voir Annexe 1).

\footnotetext{
${ }^{5}$ Il s'agit respectivement de: Journal of Business Venturing (JBV); Entrepreneurship Theory and Practice (ETP); Small Business Economics (SBE); Journal of Small Business Management (JSBM);International Small Business Journal (ISBJ); Entrepreneurship and Regional Development (ERD);Strategic Entrepreneurship Journal (SEJ) pour les revues qualifiées "entrepreneuriat" et de Academy of Management Journal (AMJ), Academy of Management Review (AMR), Administrative Science Quaterly $(A S Q)$, Strategic Management Journal (SMJ) pour les revues qualifiées "généralistes".

${ }^{6}$ La Revue des Sciences de Gestion est indexée également sur Ebsco, mais ce n'est pas le cas pour les autres revues francophones.
} 


\section{La motivation entrepreneuriale des femmes}

Privilégiant à l'origine une vision de nature économique, la littérature en entrepreneuriat a longtemps développé l'idée que la décision de démarrer une entreprise était le résultat d'un processus de maximisation dans lequel l'individu compare les revenus potentiels issus des différentes activités et choisit la solution la plus rentable (Arenius et Minniti, 2005). Cette conception a permis la diffusion de nombreux travaux et notamment ceux que l'on qualifie d'approche «push/pull» (Shapero, 1975, Shapero et Sokol, 1982 ; Vesper, 1980). En effet, suivant cette approche, un individu va se tourner vers l'entrepreneuriat si cette option lui permet un meilleur rendement économique, soit dans le cas d'un manque d'emploi (push), soit parce qu'il espère gagner davantage (pull). L'intérêt de cette approche, bien connue maintenant, est qu'elle permet de faire une distinction entre des entrepreneurs «par nécessité » et des entrepreneurs «par opportunité ». Ainsi, suivant Shapero (1975), Shapero et Sokol (1982) et Vesper (1980), un individu peut être attiré par la création d'entreprise par une opportunité de marché ou à cause d'une nécessité, comme un changement (négatif) dans sa vie, tel que le chômage ou l'immigration.

Pour autant, la recherche montre également que d'autres raisons, telles que le besoin de réalisation, la possibilité de créer son propre emploi, ou le désir d'autonomie, sont souvent au premier rang des facteurs de motivation (Verstraete et Saporta, 2006), reléguant alors l'idée de maximisation des bénéfices en arrière-plan (Carsrud et Brannback, 2011). Malgré tout, l'approche push/pull reste encore la plus utilisée dans les études de motivation entrepreneuriale (Williams et Williams, 2012 ; Eijdenberg et Masurel, 2013), et sert notamment de base aux enquêtes du Global Entrepreneurship Monitor (GEM).

Dans le cas des femmes qui entreprennent, l'approche push/pull a été également utilisée (Orhan et Scott, 2001 ; McGowan et al., 2012). Les recherches, qui s'étalent entre 1997 et 2011, montrent une certaine stabilité des facteurs motivationnels au travers du temps. En effet, sont retenus comme facteurs motivationnels push : le manque de travail et la nécessité économique, la frustration au travail, le plafond de verre ainsi que le manque de structures de garde d'enfants. Pour Ducheneaut (1997), les facteurs push qui motivent les femmes sont l'insatisfaction au travail (plafond de verre inclus), la nécessité économique et le besoin de flexibilité. De leur côté, Moult et Anderson (2005) mettent en avant l'insuffisance des revenus, l'insatisfaction dans le travail, le chômage et la nécessité d'équilibrer les responsabilités professionnelles et familiales.

Concernant les facteurs motivationnels pull, les auteurs s'accordent principalement sur leur caractère intrinsèque comme : le désir d'indépendance, le besoin de réalisation, l'attrait de la richesse ou du statut. Ainsi, Holmen et al. (2011) mettent en avant la découverte d'une opportunité d'affaires, le désir d'indépendance, le désir d'épanouissement personnel, la réalisation de soi et une plus grande satisfaction dans le travail. Il en est de même pour Jennings et Brush (2013) pour qui les facteurs pull se manifestent par le désir d'indépendance, d'épanouissement personnel, et la recherche de revenus. 
Les principales motivations repérées par les auteurs en entrepreneuriat féminin ont été récapitulées dans le tableau 1.

Tableau 1 : Principaux facteurs de motivation entrepreneuriale féminine

\begin{tabular}{|c|c|}
\hline Auteurs & Principaux Facteurs Motivationnels des Femmes \\
\hline Hisrich (1986) & $\begin{array}{l}\text { Désir d'indépendance, de réalisation, de satisfaction au travail, } \\
\text { d'accomplissement de soi, d'indépendance financière, de combler } \\
\text { l'ennui d'être une femme au foyer, réponse à une nécessité } \\
\text { économique }\end{array}$ \\
\hline Buttner et Moore (1997) & $\begin{array}{l}\text { Désir de relever un défi, d'autodétermination, de conciliation travail- } \\
\text { famille, réponse à une frustration face aux possibilités d'avancement } \\
\text { de carrière, à la discrimination }\end{array}$ \\
\hline Tzemah (2000) & $\begin{array}{l}\text { Développement personnel, nécessité économique, désir de } \\
\text { réalisation, sur les conseils d'amis ou de la famille, haut degré } \\
\text { d'estime, besoin de créativité }\end{array}$ \\
\hline Hughes (2006) & $\begin{array}{l}\text { Désir d'indépendance, de liberté, d'être son propre patron, de relever } \\
\text { un défi, d'opportunité financière, d'équilibre travail-famille, } \\
\text { d'horaires flexibles, réponse au chômage, possibilité de travailler à } \\
\text { domicile, possibilité de gagner plus d'argent, reconnaissance d'un } \\
\text { travail rémunéré }\end{array}$ \\
\hline Simonin (2006) & $\begin{array}{l}\text { Désir d'indépendance, d'accomplissement de soi, passion pour } \\
\text { l'initiative, envie de générer des revenus, d'un statut social, d'exercer } \\
\text { un pouvoir, pour palier un faible revenu familial, en réponse à une } \\
\text { insatisfaction de salaire, à des difficultés à trouver un emploi, } \\
\text { meilleure conciliation travail-famille }\end{array}$ \\
\hline Humbert, Drew (2010) & $\begin{array}{l}\text { Désir d'indépendance, recherche de satisfaction, désir de relever un } \\
\text { défi, opportunité de marché, envie de gagner plus d'argent, } \\
\text { conciliation travail-famille, rêve d'être entrepreneur }\end{array}$ \\
\hline Davis, Abdiyeva (2012) & $\begin{array}{l}\text { (En contexte d'économie en développement) } \\
\text { Se créer son propre emploi, besoin de trouver des revenus pour } \\
\text { échapper à la pauvreté, besoin de subsistance }\end{array}$ \\
\hline McGowan et al. (2012) & $\begin{array}{l}\text { Désir de relever un défi, de s'accomplir, d'être son propre patron, } \\
\text { d'avoir une meilleure conciliation entre vie professionnelle et } \\
\text { personnelle, insatisfaction au travail }\end{array}$ \\
\hline
\end{tabular}

Comme on le constate, les différents facteurs se maintiennent de façon stable au cours $\mathrm{du}$ temps. Pour autant, des divergences apparaissent chez les auteurs dans l'ordonnancement et/ou le classement parmi les facteurs de motivation cités comme principaux par les femmes. Davis et Abdiyeva (2012), dans leur revue de littérature sur la motivation entrepreneuriale des femmes, montrent que, même si les facteurs sont stables, certains articles considèrent que les femmes sont motivées plutôt par des facteurs push, alors que d'autres considèrent plutôt des facteurs pull ou aussi des facteurs mélangés push/pull (Holmen et al., 2011 ; Brush, 1990 ; Moult et Anderson, 2005 ; McGowan et al., 2012 ; Coughlin et Thomas, 2002). Par exemple, la conciliation travail-famille, considérée traditionnellement un facteur de motivation entrepreneuriale féminin, s'avère un facteur important de motivation pour certains groupes de femmes, alors qu'elle n'est pas le moteur principal pour d'autres groupes de femmes (Buttner et Moore, 1997 ; Morris et Coxeter, 1995 ; Konrad et Langton, 1991). De cette manière, et comme le montre Shelton (2006), les femmes créatrices vont établir différentes stratégies pour contrôler l'équilibre travail-famille; certaines femmes sont plus intéressées par le travail, et d'autres davantage par la famille. 
Des recherches supplémentaires présentent l'idée que les femmes entreprennent plus par volonté que par nécessité, en fonction du style de vie qu'elles désirent et par rapport aux arrangements familiaux (Hakim, 2000). Mais selon Cornet et Constantinidis (2004) et Kirkwood (2009), le plafond de verre est un important facteur push de motivation. Finalement, pour Jennings et Brush (2013), il y aurait suffisamment de preuves pour dire que les femmes sont principalement motivées par la nécessité. Néanmoins, au-delà de la stabilité de facteurs motivationnels chez les femmes, la variabilité dans l'ordonnancement de ces facteurs nous amène à penser à une diversité de profils d'entrepreneures, au croisement des caractéristiques internes (niveau d'éducation, désirs...) et externes (possibilités d'emploi, cycle de vie de la famille...).

\section{La diversité d'entrepreneures au travers des principales typologies fondées sur les motivations}

Tant que les recherches se focalisaient sur la question de savoir si l'entrepreneuriat féminin était un entrepreneuriat différent ou similaire à l'entrepreneuriat masculin, les entrepreneures étaient plutôt considérées comme un groupe homogène.

Il faut attendre les années 2000, pour constater que certains auteurs commencent à investiguer la diversité des femmes qui entreprennent en s'appuyant principalement sur l'étude des facteurs motivationnels. A l'issue de cette analyse, ils proposent les premières typologies d'entrepreneuriat féminin. Par exemple, Bruni, Gherardi, et Poggio (2004) présentent sept groupes de femmes : celles cherchant un emploi suite au chômage, celles considérant l'entrepreneuriat comme une stratégie principale de long terme, celles qui cherchent à contourner le problème de plafond de verre, celles qui cherchent la conciliation travail-famille ainsi que celles qui réintègrent la vie active après avoir quitté leur emploi pour s'occuper de leur famille, celles qui suivent une tradition familiale entrepreneuriale et finalement, la femme féministe.

Morris et al. (2006), pour leur part, classent les entrepreneures en deux groupes au regard de leurs motivations à la croissance de leur entreprise : celles qui sont motivées par une croissance forte (ou « high growth entrepreneurs ») et celles qui visent plutôt une croissance modeste (ou «modest growth entrepreneurs »). Les premières sont motivées par des facteurs pull, comme le désir d'être riche ou de relever un défi. Les deuxièmes se définissent plutôt par des facteurs push, comme la possibilité d'avoir un revenu suffisant pour assurer la sécurité financière de leur famille, préférant contrôler la croissance de leur entreprise à des niveaux compatibles avec leur style de vie et les besoins de la famille. Bien que les deux groupes de femmes s'accordent sur le fait que les exigences familiales agissent comme un facteur de croissance, chaque groupe les hiérarchise différemment. Pour le premier groupe, l'entreprise " passe devant » et c'est le prix à payer pour assurer la croissance de l'entreprise, alors que pour le second groupe, c'est « la famille d'abord», expliquant un développement plus modeste de l'entreprise. Par la suite, Lewis (2013) reprend cette distinction et ajoute une troisième catégorie en reconnaissant trois profils d'entrepreneures : celles orientées famille, celles orientées croissance et celles qui ne cherchent pas la croissance. La littérature signale cependant que des fenêtres d'opportunité de croissance s'ouvrent à nouveau pour les 
femmes matures au fur et à mesure que les enfants grandissent et que les exigences d'éducation se réduisent (Moult et Anderson, 2005).

De son coté, Hughes propose, dans son article paru en 2006, de classer les femmes suivant leurs motivations également en trois groupes : l'entrepreneure classique (c'est-àdire sans que la distinction sexuée ou genrée n'interfère pas dans la nature de la démarche entrepreneuriale), la femme qui arbitre entre son travail et sa famille et l'entrepreneure forcée c'est-à-dire qui n'a d'autre choix que la création d'entreprise pour s'assurer un emploi. Dans la même lignée, Acs et al. (2005) voient l'entrepreneuriat féminin comme une possibilité d'insertion dans l'emploi (face à la discrimination) et aussi comme une manière de combiner les rôles productifs et reproductifs des femmes. Cette classification se retrouve d'ailleurs du coté francophone avec la classification développée par Le Loarne-Lemaire et al. (2012). Les auteures distinguent six profils de femmes : la femme qui entreprend après avoir eu des enfants, celle qui entreprend après avoir été au chômage, l'étudiante entrepreneur, la femme cadre à la recherche de plus de temps pour soi, la femme aventurière à la recherche d'un nouveau défi et la «business women » qui entreprend après la cinquantaine.

Constantinidis et al. (2006) considèrent les femmes qui entreprennent comme un groupe hétérogène, avec des réalités multiples. Les femmes sont ainsi différentes en leurs caractéristiques personnelles, le type d'entreprise qu'elles vont créer et le type de financement qu'elles vont chercher. Les auteurs, en utilisant des théories de capital humain et social, identifient trois types d'entrepreneures : celles avec un haut niveau de capital humain, social et financier; celles avec un bas niveau de capital humain, social et financier; et celles qui vont reprendre une affaire familiale.

Comme le montre l'analyse des différents articles, il y a lieu de s'interroger sur la multiplicité des profils d'entrepreneur féminin en lien avec leurs facteurs de motivation et le cycle de vie (productif ou reproductif) des femmes. En effet, pour Moult et Anderson (2005), certains facteurs motivationnels vont être plus en rapport avec les périodes de la vie de la femme. Par exemple, lorsque les responsabilités familiales sont à leur apogée, la recherche d'une certaine flexibilité entre vie professionnelle et vie familiale a un rôle important pour les femmes. Cependant, il semble raisonnable de penser que lorsque les enfants grandissent, d'autres facteurs seront plus importants pour les entrepreneures. 
Tableau 2 : Principales typologies d'entrepreneuriat féminin

\begin{tabular}{|l|l|}
\hline Auteurs & Typologie \\
\hline Bruni, Gherardi et & Aimless (pour sortir du chômage) \\
& Success oriented (comme une stratégie de long terme) \\
& Strongly success oriented (pour l'épanouissement et contre le plafond de verre) \\
Dualists (équilibre travail-famille) & Return workers (après la maternité) \\
Traditionalists (tradition familiale entrepreneuriale) & Radicals (féministes) \\
\hline Morris et al. (2006) & $\begin{array}{l}\text { High growth entrepreneurs } \\
\text { Modest growth entrepreneurs }\end{array}$ \\
\hline Hughes (2006) & $\begin{array}{l}\text { Classic entrepreneurs } \\
\text { Forced entrepreneurs } \\
\text { Work-family entrepreneurs }\end{array}$ \\
\hline $\begin{array}{l}\text { Constantinidis et al. } \\
\text { (2006) }\end{array}$ & $\begin{array}{l}\text { Entrepreneures avec un haut niveau de capital social, humain et financier } \\
\text { Entrepreneures avec un bas niveau de capital social, humain et financier } \\
\text { Femmes qui reprennent une affaire familiale }\end{array}$ \\
\hline $\begin{array}{l}\text { Le Loarne-Lemaire et } \\
\text { al. (2012) }\end{array}$ & $\begin{array}{l}\text { Entrepreneure après maternité } \\
\text { Entrepreneure après chômage } \\
\text { Etudiante entrepreneur } \\
\text { Femme en recherche de temps pour soi } \\
\text { Femme aventurière } \\
\text { Business women }\end{array}$ \\
\hline Lewis (2013) & $\begin{array}{l}\text { Family-oriented entrepreneurs } \\
\text { Growth-oriented entrepreneurs } \\
\text { Non-growth-oriented entrepreneurs }\end{array}$ \\
\hline
\end{tabular}

\section{Identification de trois idéaux-types dans la recherche en entrepreneuriat féminin}

A partir d'un recueil d'articles en motivation entrepreneuriale féminine, et en analysant les principales typologies d'entrepreneuriat féminin, nous avons repéré une certaine récurrence de profils qui a abouti à l'identification de trois figures ou idéaux-types (au sens de Weber) qui apparaissent majoritairement dans les recherches et au cours du temps : un entrepreneuriat par nécessité, un entrepreneuriat par opportunité ou (transition) de carrière, et un entrepreneuriat par conciliation travail-famille qui intègre la maternité des femmes.

\subsection{Les femmes qui entreprennent par nécessité}

De nombreuses études se sont focalisées sur les femmes qui entreprennent par nécessité par opposition aux femmes qui entreprennent par opportunité, nous identifions cette première catégorie d'entrepreneure comme un des trois idéaux-types fondamentaux. L'entrepreneuriat féminin par nécessité a été considéré dans les typologies de Bruni, Gherardi et Poggio (2004) ; Hughes (2006), ou aussi Le Loarne-Lemaire et al. (2012).

Comme pour les hommes, les femmes qui entreprennent par nécessité agissent principalement pour des raisons économiques. Leurs motivations reposent essentiellement sur des facteurs push, à savoir : vouloir assurer leur subsistance, pallier une situation de chômage ou l'impossibilité de trouver un emploi, ou encore contourner des pratiques de discrimination ou de marginalisation. Dans ces conditions, 
l'entrepreneuriat par nécessité (aussi bien des hommes que des femmes) représente un moyen pour assurer la satisfaction des besoins primaires de l'individu (au sens de Maslow, 1954). Cependant, comparées aux hommes, les femmes ont plus de barrières face à l'emploi. Elles sont, comme le montre une étude de l'Organisation Internationale du Travail (ILO, 2004), les dernières à être embauchées et les premières à être licenciées (Elam et Terjesen, 2010). Aussi sont-elles contraintes de créer leur entreprise en raison d'un manque d'emploi ou d'un emploi non satisfaisant et pour lequel la rémunération n'est souvent pas suffisante pour assurer la survie de l'individu ou de son groupe familial primaire.

Certaines recherches montrent qu'il y a plus d'entrepreneures dans les pays en développement que dans les pays développés (Minniti et al., 2006). Une part de ce résultat peut sans doute s'expliquer par la difficulté pour les femmes de trouver un emploi dans des pays où culturellement et soci(ét)alement elles sont reléguées à des activités domestiques. Souvent moins instruites, elles trouvent le moyen de s'insérer dans l'économie locale au travers de l'entrepreneuriat (Ascher, 2012; Langowitz et Minniti, 2007, Torri et Martinez, 2014). Aussi, Verheul et al. (2006) estiment-ils qu'il y a plus de femmes qui entreprennent dans l'économie informelle dans les pays en voie de développement. Notamment dans les pays pauvres et peu industrialisés, nombreuses sont les femmes qui se consacrent à la production alimentaire à petite échelle, comme une manière de subvenir aux besoins de leurs familles. C'est le cas par exemple de l'Ouganda (Snyder, 2003), où $80 \%$ de toute la nourriture cultivée est produite par des femmes (Davis et Abdiyeva, 2012). Le soutien et le développement d'un tel type d'entrepreneuriat féminin est un objectif nécessaire pour réduire la pauvreté et permettre aux femmes de se développer (Minniti et Naude, 2010).

Plus généralement, le nombre croissant de femmes participant à des activités entrepreneuriales serait un indicateur du niveau de transformation dans les domaines économiques et soci(ét)aux des pays (Holmen et al., 2011 ; Ascher, 2012). Il indiquerait que les femmes seraient en train d'acquérir une plus grande autonomie et une confiance dans leurs compétences et leurs capacités à construire des entreprises à égalité de succès, voire plus, que les hommes. Dans les pays en voie de développement, l'entrepreneuriat féminin pourrait être favorisé par les possibilités de financement au travers des agences de microcrédit et des dynamiques familiales qui permettent la collaboration des membres de la famille dans le déroulement du projet entrepreneurial (Welsh et al., 2013). Tout en favorisant l'autonomisation des femmes, l'accès à la micro-finance améliore leur niveau d'aspirations, diminue les risques de la création et réduit la marginalisation. Cependant, les résultats des études menées en Afrique, montrent que l'accès à la microfinance n'a pas permis une amélioration dans la qualité de vie de ces femmes : elles continuent à vivre dans un niveau de subsistance, sans croissance des entreprises créées (Welsh et al., 2013).

Certains auteurs partagent l'idée que les femmes des pays en voie de développement seraient plus tournées vers la création par nécessité, contrairement aux femmes des pays développés plus enclines à créer des entreprises par opportunité (Langowitz et Minniti, 2007 ; Brush et Cooper, 2012) Néanmoins, ceci doit être reconsidéré en prenant en compte l'évolution des situations économiques et soci(ét)ales des pays. Par exemple, plusieurs études menées en Grande-Bretagne montrent l'existence d'un entrepreneuriat 
féminin par nécessité économique à partir des années 1990 (Hughes, 2003). Coughlin et Thomas (2002) l'expliquent comme étant consécutif, d'une part, à l'augmentation des divorces et des familles monoparentales, et d'autre part, aux politiques de restructuration des entreprises et à l'élimination des postes qui étaient destinés aux femmes. Par conséquent, la responsabilité de devoir assurer la subsistance de son groupe familial pousse la femme vers l'entrepreneuriat. Ainsi, l'entrepreneuriat par nécessité est directement lié au cycle de vie de la femme et de sa famille, en plus de sa relation au niveau de développement du pays,

\subsection{Les femmes qui choisissent une (transition de) carrière entrepreneuriale}

Un autre groupe de recherches s'est intéressé aux femmes qui, possédant déjà un emploi, décident de créer une entreprise. Les raisons peuvent être liées à l'insatisfaction au travail, plafond de verre inclus, ou à la recherche de l'épanouissement au travers d'une carrière entrepreneuriale. Ces femmes représentent un deuxième idéal-type, celui de la femme choisissant l'entrepreneuriat comme une (transition de) carrière.

Parmi la variété des profils d'entrepreneurs féminins se distingue un important groupe de femmes qui créent des entreprises en étant motivées par le désir de relever un défi, d'exprimer leur créativité, de rechercher leur indépendance et l'autonomie, de trouver une opportunité, etc. Autrement dit, dès lors que sont pris en compte des critères d'appréciation plus subjectifs, les femmes qui entreprennent sont motivées principalement par des facteurs pull - se plaçant dans le haut de l'échelle des besoins (au sens de Maslow, 1954).

Dans l'étude menée par Hughes (2006) au Canada, plus de la moitié de femmes interrogées se classent dans cette catégorie, que l'auteur appelle des entrepreneures classiques. Dans ce type d'entrepreneuriat féminin se reconnaissent des femmes qui, de manière volontaire et sans être poussées par les circonstances, ont choisi de créer leur entreprise. Certaines vont considérer l'entrepreneuriat directement comme une carrière, en créant des entreprises dès leur sortie d'études, d'autres vont penser à l'entrepreneuriat comme une continuation dans leur carrière professionnelle. Les recherches qui s'appuient sur les logiques de carrière entrepreneuriale mettent l'accent sur le rôle central de l'individu dans la construction de son parcours professionnel (Bowen et Hirish, 1986). Cherchant à se réaliser sur plusieurs fronts, tant dans la sphère économique que dans la sphère familiale ou personnelle (Brush, 1992 ; Le LoarneLemaire et al., 2012), les femmes répondent aux transformations économiques et sociales du marché du travail en se construisant des carrières moins linéaires et plus discontinues que les hommes, voire « sinusoïdales » selon l'expression de Lebègue (2015: 98).

Cette catégorie d'entrepreneures permet de regrouper tous les cas des femmes qui ne sont pas dans une création par nécessité économique ou par conciliation travail-famille comme principal motif. Ainsi, des profils variés tels que l'étudiante entrepreneur, la femme aventurière ou la Business women de la typologie de Le Loarne-Lemaire et al. (2012) vont partager des caractéristiques motivationnelles. De la même manière, l'entrepreneure classique de Hughes (2006) et plusieurs catégories de Bruni, Gherardi et 
Poggio (2004), tels que les femmes « (strongly) success oriented », « traditionalists » ou « radicals », peuvent se regrouper dans cette classification.

Pour les femmes qui choisissent l'entrepreneuriat comme premier choix de carrière, la littérature met en avant leur éducation et leur formation avec des niveaux d'études postsecondaires et universitaires (Hughes, 2006 ; Gatewood et al., 2009), et des niveaux d'éducation supérieurs à celui des hommes entrepreneurs dans les pays développés (Langowitz et Minniti, 2007). Dans l'ensemble, ces entrepreneures d'un type «classique » aspirent à créer des entreprises à forte croissance et nécessitant un financement extérieur (Gatewood et al., 2009).

Pour les femmes qui choisissent la carrière entrepreneuriale après avoir eu une expérience préalable de travail salarié, leur choix suit une logique de continuité et non de rupture. Comme le montrent Buttner et Moore (1997) à partir d'une étude de 129 femmes ayant démissionné de leur poste de direction pour créer leur propre entreprise, deux motivations principales ont été observées. D'une part un désir de relever un défi et d'être indépendante, et d'autre part un besoin de trouver un équilibre entre leurs vies professionnelles et familiales. De longues heures au travail, ainsi qu'un manque de flexibilité peuvent mener les femmes qualifiées à choisir l'auto-emploi comme le montrent Anderson et al. (2010). Cependant, d'autres études effectuées sur les femmes cadres montrent que même si l'équilibre travail-famille est important, l'influence que cela portait dans les choix de carrière des femmes n'était pas différente que dans le cas des hommes (Konrad et Langton, 1991 ; Morris et Coxeter, 1995).

S'il est vrai que pour chacune de ces femmes le choix est perçu comme volontaire, certaines d'entre elles abordent l'entrepreneuriat comme une manière de contourner la frustration causée par une certaine discrimination dans le cadre de l'emploi. Cette discrimination peut prendre une forme évidente (comme la non progression dans l'échelle hiérarchique et de responsabilités : cas du plafond de verre) ou plus subtile car moins aisément décelable (cas des différences de salaire homme/femme à des postes similaires). Dès lors, parmi les différentes formes de discrimination, le plafond de verre est, plus spécifiquement pour les femmes qualifiées, un moteur de l'entrepreneuriat (Hisrich et Brush, 1985 ; Coughlin et Thomas, 2002 ; Cornet et Constantinidis, 2004 ; Kirkwood, 2009) et ce, indépendamment du niveau de développement des pays (Davis et Abdiyeva, 2012). Ces recherches viennent confirmer l'étude menée par Cromie (1987) qui montrait déjà que les femmes (et aussi les hommes) étaient motivées principalement par un désir d'autonomie, de réalisation de soi et de satisfaction au travail et que la réalité du plafond de verre était avérée dans le cas des femmes qualifiées puisque la plupart des hauts postes étaient occupés par des hommes. Par conséquent, l'entrepreneuriat féminin comme transition de carrière est une solution satisfaisante pour faire face à cette réalité, et ce d'autant plus pour les femmes qualifiées qui peuvent espérer un meilleur revenu que lorsqu'elles étaient en situation d'emploi en tant que salariées (Elam et Terjesen, 2010). 


\subsection{Les femmes en recherche d'équilibre travail-famille et la maternité : les cas des mampreneurs}

Même si l'équilibre travail-famille a été largement traité dans le cas du travail des femmes, une littérature grandissante a vu le jour depuis quelques années, identifiant la figure de mampreneur : maman et entrepreneur. Cette profusion d'articles nous permet de considérer un troisième idéal-type. Celui-ci a été décrit comme «dualist» dans la typologie de Bruni, Gherardi et Poggio (2004), ou «travail-famille » pour Hughes (2006) et Lewis (2013).

Bien que non niée par les chercheurs en entrepreneuriat féminin, l'incidence de la maternité dans les motivations entrepreneuriales a pendant longtemps été en quelque sorte occultée par celles légitimes concernant l'arbitrage et la recherche d'un équilibre entre le travail et la famille. Comme l'indiquent Le Loarne-Lemaire et al. (2012) dans leur livre au titre évocateur «Femme et entrepreneur, c'est possible », certaines femmes se lancent dans la création de leur entreprise afin de concilier vie familiale et vie personnelle, alors que d'autres visent la satisfaction et l'accomplissement de soi. Ainsi, les résultats des études sur la motivation entrepreneuriale des femmes montrent une diversité de facteurs permettant de classer les femmes dans des catégories à la croisée de leur cycle de vie de femme et de leur trajectoire ou carrière professionnelle en fonction de leurs besoins.

Pour Cromie (1987), l'entrepreneuriat est un moyen pour les femmes de répondre simultanément à leurs propres besoins de carrière et à ceux de leurs enfants. Dans son étude, les répondantes étaient des jeunes femmes ayant une famille, dont un certain nombre avait renoncé à un emploi sûr et bien rémunéré pour créer leur entreprise. La création d'entreprise permet plus de flexibilité en termes d'heures et de lieu de travail en concordance avec les besoins des enfants. C'est aussi ce que souligne Simonin (2006) qui stipule que les femmes choisissent une carrière entrepreneuriale en raison de l'autonomie et de la souplesse qu'elles y trouvent. Peu satisfaites de leur carrière en entreprise, elles perçoivent l'entrepreneuriat comme un outil pour ajuster leur carrière à leurs «devoirs » familiaux. Selon Hugues (2006), ce groupe d'entrepreneures qu'elle désigne comme «entrepreneures travail-famille » comprend les personnes qui citent : «l'équilibre travail-famille», les « horaires flexibles », « la possibilité de travailler à la maison » comme principaux facteurs de motivation. En termes d'âge, ce groupe de femmes diffère de celui des entrepreneures «classiques » plus âgées. Il serait concentré à mi parcours de la vie (résultat assez prévisible) et composé de femmes mariées ou vivant en union libre.

Par conséquent, l'incidence de la maternité sur les facteurs de motivations entrepreneuriales doit être regardée de plus près par les chercheurs. Dans une recherche récente, McGowan et al. (2012) rappellent que si les grandes entreprises ont mieux pris en compte la problématique des conflits inhérents aux horaires de travail classiques $(9 \mathrm{~h} / 17 \mathrm{~h})$ en proposant des horaires plus flexibles ou la possibilité d'effectuer des heures de travail à la maison, il reste que la vie de famille a un impact négatif sur l'avancement des femmes cadres avec jeunes enfants. Citant Winn (2004: 144) : "Comme la culpabilité et le stress dépassent les avantages de revenus et de prestige, les femmes avec de jeunes enfants sont de plus en plus à quitter la population active pour devenir 
mères à temps plein » et « comme les exigences de la vie des entreprises empiètent sur la qualité et la quantité de temps passé en famille», certaines femmes choisissent de ne pas redevenir employées et préfèrent créer leur propre entreprise, afin d'avoir un meilleur équilibre entre travail et famille.

Brush et al. (2009) proposent une conceptualisation qui intègre les différentes dimensions qui impactent le projet entrepreneurial des femmes. Elles distinguent les contextes qui influencent leurs actions et elles prennent en compte leur encastrement social (modèle en $5 \mathrm{M}$ ). Ce modèle s'appuie en premier lieu sur trois dimensions principales considérées comme les fondements de la création d'entreprise : le marché (Market), l'argent (Money) et la gestion de l'entreprise (Management). Le marché correspond à l'accès aux affaires et, lors de la création d'une nouvelle entreprise, à l'accès à des opportunités ; l'argent renvoie à l'accès et à la disponibilité du capital financier; et le management se rapporte au capital humain et à l'organisation (Ettl et Welter, 2010). Elles l'enrichissent en second lieu avec deux dimensions : le méso/macro environnement et la maternité, qui ont un impact majeur sur l'activité entrepreneuriale des femmes. Concernant la dimension méso/macro de l'environnement, les auteures insistent sur la nécessaire prise en compte des enjeux et des contraintes qui vont au-delà du marché, telles que les attentes de la société et les normes culturelles (au niveau macro) et celles des institutions intermédiaires et régionales (au niveau méso). Concernant la dimension de la maternité, définie comme une métaphore du contexte ménage/famille, elles conçoivent la place de la femme entrepreneur en position de «membre du collectif » par rapport à la fonction de maximisation de l'utilité conjointe du ménage ${ }^{7}$.

Grâce à la contextualisation (Zahra, 2007 ; Welter, 2011), les auteures introduisent un rééquilibrage entre l'influence de facteurs externes (macro et méso environnement) et la perception de facteurs internes ou personnels (microenvironnement), ce qui est le cas pour les entrepreneures et mères de jeunes enfants. Le contexte de la maternité devient alors propice à l'expression de motivations entrepreneuriales des femmes dans une perspective d'encastrement familial (Aldrich et Cliff, 2003), dans laquelle l'environnement familial va façonner la création d'entreprise et vice-versa.

Dans le même temps, une littérature grandissante se développe autour des mampreneurs considérées comme de nouvelles figures en entrepreneuriat féminin (d'Andria, 2014). Derrière ce néologisme, qui signifie maman et entrepreneur (Cobe et Parlapiano, 2002), il s'agit de prendre en compte une nouvelle réalité d'entrepreneuriat où l'entreprise et l'enfant sont conçus de manière presque simultanée. S'identifiant à la fois comme mère et femme d'affaires, la mampreneur décide d'impulser une nouvelle organisation dont elle est propriétaire et gestionnaire, afin d'atteindre un équilibre professionnel et privé difficile, voire impossible dans le salariat, et dont l'opportunité d'affaires est généralement en lien avec l'expérience particulière d'avoir des enfants (RichommeHuet et al., 2013).

\footnotetext{
7 "In an attempt to provide an extended household perspective neoclassical economic analysis, for instance, has moved from the individual to fit in the family by assuming utility maximization of a joint utility function and simple optimality of household division of labor and distribution of income" (Brush et al., 2009: 10).
} 
D'après Korsgaard (2007), la principale motivation de la mampreneur est de pouvoir concilier sa vie professionnelle et familiale afin de trouver un équilibre entre son lieu de travail ou de carrière et les besoins de sa propre famille, tout en combinant une expérience de travail intéressante. De même, Jean et Forbes (2012) montrent que les motivations entrepreneuriales de mampreneurs canadiennes de la province de l'Ontario relèveraient exclusivement de facteurs de nature push. A partir de l'étude de vingt cas, elles constatent que les mampreneurs entreprennent afin de pouvoir combiner leur projet personnel avec leur vie de famille. En revanche, l'étude de Nel et al. (2010) à partir de trois études de cas approfondies de mampreneurs australiennes indique que les mampreneurs sont motivées aussi bien par des facteurs push que pull, tout en recherchant comme premier objectif un meilleur environnement pour leurs familles et l'ensemble de la communauté. Les auteurs identifient l'équilibre entre vie professionnelle et familiale, l'envie de se réaliser, la satisfaction personnelle, l'augmentation de revenu, la possibilité de gagner en respect sur la question de l'égalité des sexes et le fait de devenir indépendante comme des facteurs de motivation entrepreneuriale pour les mampreneurs.

De son coté, une étude d'Ekinsmyth (2011) souligne l'importance de la prise en compte de l'incidence des critères géographiques et économiques sur les pratiques entrepreneuriales des mampreneurs. L'étude se fonde sur une analyse du bassin économique des West Midlands au sud de l'Angleterre et met en relation le contexte dégradé de l'emploi et la forte augmentation du taux de chômage à Birmingham depuis les années 1990. L'auteure montre alors que les mampreneurs sont un sous-groupe de femmes entrepreneures situées à l'interface entre le monde du travail rémunéré et la maternité. Complétant cet éclairage, Duberley et Carrigan (2012) montrent que des mampreneurs de la même région se lancent dans la création d'entreprise au moment de la venue d'un enfant pour devenir indépendantes et échapper à l'idée d'être perçues comme des mères au foyer. Ces résultats viennent confirmer l'intérêt du modèle de Brush et al. (2009) d'intégrer les dimensions du méso/macro environnement et de la maternité.

\section{Conclusion et perspectives}

Façonné au rythme des mutations du capitalisme, l'entrepreneuriat évolue (Boutillier et Uzunidis, 1999, 2015 ; Hernandez, 2006) et avec lui la motivation des entrepreneurs (hommes et femmes). Construit complexe, la motivation peut varier et se modifier avec le temps et les circonstances (Williams et Williams, 2012), combinant des aspects internes à la personne (autant psychologiques que culturels) et externes (environnement économique et institutionnel, mesures politiques...).

L'objectif de cet article était de revenir sur les trente dernières années de recherches sur les motivations entrepreneuriales des femmes en mettant en avant les apports successifs. A partir d'un recueil étendu de la littérature, trois idéaux-types d'entrepreneures peuvent être aujourd'hui retenus. 
Le premier groupe est constitué de femmes qui entreprennent parce qu'elles n'ont pas d'autres choix. Cet entrepreneuriat féminin par nécessité, majoritaire dans les pays en voie de développement, s'identifie avec les besoins de survie et de sécurité et s'appuie sur des facteurs push de motivation. Ces femmes sont jeunes, avec ou sans enfants, et ont des difficultés pour trouver un emploi ou un emploi satisfaisant. Elles doivent assurer leur subsistance et celle de leur groupe familial. Pour autant, cet entrepreneuriat de nécessité (ou par défaut) n'est pas l'apanage de pays en développement ou en transition économique. Cette tendance se retrouve également dans les pays considérés comme économiquement plus avancés. Pour Boutillier et Uzunidis (2015), le développement de l'entrepreneuriat est devenu depuis une vingtaine d'années un instrument des pouvoirs publics en direction de l'insertion professionnelle des populations les plus éloignées du marché du travail comme notamment les femmes. Ils soulignent que «toutes les catégories sociales dont l'insertion professionnelle peut poser (ou pose) problème disposent d'une solution : la création d'entreprise, qui tend à devenir, dans l'esprit des politiques, une planche de salut tout à fait acceptable pour des populations qui non seulement cumulent les handicaps sociaux (sexe, âge - jeunes ou âgés - absence de diplômes, etc.), mais également des individus qui au contraire - en raison de leur haut niveau de qualification - sont devenus trop chers et par conséquent inaptes à l'entreprise ou à la recherche publique » (Boutillier et Uzunidis, 2015 : 29).

Parallèlement, d'autres femmes sont dans la recherche d'autoréalisation et de satisfaction au travail. Nous les avons caractérisées comme des femmes entrepreneurs de carrière ou en transition vers une carrière entrepreneuriale. Loin d'être exclues du marché du travail, elles trouvent leur réalisation dans l'entrepreneuriat. Elles se placent en haut de la hiérarchie des besoins (au sens de Maslow, 1954) et correspondent généralement aux femmes qui n'ont pas de problème de conciliation entre leur vie au travail et leur vie de famille, ou en tout cas pas plus que les hommes. Elles sont principalement motivées par des facteurs pull, mais peuvent être aussi motivées par des combinaisons de facteurs push/pull.

Un troisième groupe correspond aux femmes qui voient dans l'entrepreneuriat un moyen de s'épanouir aussi bien comme professionnelle que comme mère de famille, et ainsi cherchent dans l'entrepreneuriat un moyen de réussir l'équilibre travail-famille. Ces femmes, qui se revendiquent conjointement mères avec des enfants en bas âge et entrepreneures, sont identifiées dans la littérature comme mampreneurs. Leurs motivations entrepreneuriales sont une combinaison de facteurs push et pull et la plupart démarrent une transition de carrière vers l'entrepreneuriat au moment de leur grossesse, inspirées par des opportunités d'affaires en rapport à la maternité.

Par conséquent, nos lectures en entrepreneuriat féminin et en motivation entrepreneuriale des femmes permettent de faire ressortir deux aspects importants, qui ne sont pas suffisamment considérés dans la recherche en entrepreneuriat. Le premier aspect à considérer est celui de l'hétérogénéité des femmes entrepreneurs. Aujourd'hui, l'état de la recherche ne peut pas se contenter de considérer l'entrepreneuriat féminin comme une réalité homogène. En effet, il existe (au moins) trois idéaux-types d'entrepreneures. 
Le deuxième aspect à considérer est l'interrelation entre l'entrepreneuriat et la famille. Comme le soulignent Aldrich et Cliff (2003), les questions familiales et entrepreneuriales ont souvent été séparées alors qu'elles sont la plupart du temps intriquées. En tenant compte des responsabilités productives et reproductives des femmes, les transformations familiales (relations et rôles entre les membres...) ont des conséquences sur les décisions et la création d'une entreprise. Comme le montre l'article de Ronsen (2014), ce n'est pas seulement la présence d'enfants dans la famille qu'il faut considérer en étudiant l'entrepreneuriat féminin, mais aussi la situation de travail et les caractéristiques du partenaire. En effet, c'est tout le cadre familial qui va agir sur la décision de création de la femme.

Aussi, considérons-nous que les prochaines recherches en entrepreneuriat féminin devront prendre en compte cette diversité. Pour cette raison, la recherche doit faire preuve de nuances lors de la généralisation des résultats et intégrer plus de critères de contextualisation.

\section{Références}

ACS Z., ARENIUS P., HAY M., MINNITI M. (2005), Global Entrepreneurship Monitor 2004 Executive Report, Babson College and London Business School.

AHL H. (2006), "Why research on women entrepreneurs needs new directions", Entrepreneurship Theory and Practice, vol. 30, n5, pp. 595-621.

ALDRICH H.E., CLIFF J.E. (2003), "The pervasive effects of family on entrepreneurship: Toward a family embeddedness perspective”, Journal of Business Venturing, n 18, pp. 573-597.

ANDERSON D., VINNICOMBE S., SINGH V. ( 2010) "Women partners leaving the firm: choice, what choice?", Gender in Management: An International Journal, vol. 25, n 3, pp. 170-183.

ARENIUS P., MINNITI (2005), «Perceptual Variables and Nascent Entrepreneurship», Small Business Economics, n²4, pp. 233-247.

ASCHER J. (2012), "Female Entrepreneurship - An Appropriate Response to Gender Discrimination", Journal of Entrepreneurship, Management and Innovation (JEMI), vol. 8, n 4, pp. 97-114.

BIRLEY S. (1989), "Female entrepreneurs: Are they really different?", Journal of Small Business Management, Vol.27, pp.32-37.

BOUTILLIER S., UZUNIDIS D. (1999), La légende de l'entrepreneur, Syros-La Découverte.

BOUTILLIER S., UZINIDIS D. (2015), «De la société salariale à la société entrepreneuriale ou la création d'entreprises au secours de l'emploi. Une analyse critique », La Revue des Sciences de Gestion, n²75-276, pp. 23-40.

BOWEN D., HISRICH R. (1986), "The Female Entrepreneur: A Career Development Perspective", Academy of Management Review, vol. 11, $\mathrm{n}^{\circ}$ 2, pp. 393-407.

BRUNI A., GHERARDI S., POGGIO B. (2004), "Entrepreneur - mentality, gender and the study of women entrepreneurs", Journal of Organizational Change Management, vol. 17, $\mathrm{n}^{\circ}$ 3, pp. 256268.

BRUSH C. (1990), Women and enterprise creation: An overview of women-owned business. In Enterprising Women: Local Initiatives for Job Creation. Paris: Organization for Economic CoOperation and Development (OECD).

BRUSH C. (1992), "Research on Women Business Owners: Past Trends, A New Perspective and Future Direction", Entrepreneurship Theory and Practice, vol. 16, n 4, pp. 5-30.

BRUSH C., CARTER N. M., GATEWOOD E. J., GREENE P. G., HART M. M. (2001), The Diana project: Women business owners and equity capital: The myths dispelled, Kansas City, Kauffman Center for Entrepreneurial Leadership.

BRUSH C., COOPER S.Y. (2012), «Female Entrepreneurship and Economic Development: An International Perspective», Entrepreneurship \& Regional Development: An International Journal, vol. $24, \mathrm{n}^{\circ} 1-2$, pp. 1-6. 
BRUSH C., de BRUIN A., WELTER F. (2009), «A gender-aware framework for women's entrepreneurship », International Journal of Gender and Entrepreneurship, vol. 1, n 1, pp. 8-24.

BUTTNER E.H., MOORE D.P. (1997), «Women's organizational exodus to entrepreneurship: Selfreported motivations and correlates with success», Journal of Small Business Management, vol. $35, \mathrm{n}^{\circ} 1, \mathrm{pp} .34-46$.

CARSRUD A, BRANNBACK M. (2011), «Entrepreneurial Motivations: What Do We Still Need to Know? », Journal of Small Business Management, vol.49, $\mathrm{n}^{\circ}$ 1, pp. 9-26.

CARTER S. (2000), "Improving the numbers and performance of women-owned businesses: Some implications for training and advisory services", Education \& Training, vol. 42, n 4/5, pp. 326333.

COBE P., PARLAPIANO E.H (2002), Mompreneurs. A Mother's practical Step-by-Step. Guide to Work at Home Success, Perigee Books, $2^{\text {nd }}$ ed. New York.

CONSTANTINIDIS C., CORNET A., ASANDEI S. (2006), "Financing of Women-Owned Ventures: The Impact of Gender and Other Owner- and Firm-Related Variables", Venture Capital, vol. 8, ${ }^{\circ}$ 2, pp. $133-157$.

CORNET A., CONSTANTINIDIS C. (2004), «Entreprendre au féminin. Une réalité multiple et des attentes différenciées », Revue Française de Gestion, n 151, pp. 191-204.

COUGHLIN J.H., THOMAS R.E. (2002), The rise of women entrepreneurs: People, process, and global trends, Westport: Quarum Books.

CROMIE, S. (1987), "Motivations of Aspiring Male and Female Entrepreneurs", Journal of Occupational Behavior, vol. 8, $\mathrm{n}^{\circ}$ 3, pp. 251-261.

d'ANDRIA A. (2014), «Un éclairage sur le processus entrepreneurial des mampreneurs. Etude exploratoire de leur dynamique effectuale », Revue de l'Entrepreneuriat, vol. 13, n 1, pp. 11-33.

DAVIS P., ABDIYEVA F. (2012), "En Route to a Typology of the Female Entrepreneur? Similarities and Differences Among Self-Employed Women", Journal of Management Policy and Practice, vol. 13, n 4, pp. 121-137.

De BRUIN A., BRUSH C., WELTER F. (2006), «Introduction to the special issue: towards building cumulative knowledge on women's entrepreneurship », Entrepreneurship Theory and Practice, vol. $30, \mathrm{n}^{\circ} 5$, pp. 585-594.

De BRUIN A., BRUSH C., WELTER F. (2007), « Advancing a framework for coherent research on women's entrepreneurship, Entrepreneurship Theory and Practice», Vol.31, n³, p. 323-339.

DUBERLEY J., CARRIGAN M. (2012), « The career identities of 'mumpreneurs': women's experiences of combining enterprise and motherhood », International Small Business Journal, March, vol. 0, $\mathrm{n}^{\circ} 0$, pp. 1-23.

DUCHENEAUT B. (1997), "Women entrepreneurs in SME's", OECD, Conference on Women Entrepreneurs in Small and Medium Enterprises: A Major Force for Innovation and Job Creation. Paris, France.

EIJDENBERG E., MASUREL E. (2013), «Entrepreneurial Motivation in a Least Developed Country: Push Factors and Pull Factors among Mses in Uganda», Journal of Enterprising Culture, vol. 21, $\mathrm{n}^{\circ} 1$, pp. 19-43.

EKINSMYTH C. (2011), «Challenging the boundaries of entrepreneurship: the spatialities and practices of UK 'mumpreneurs' », Geoforum, vol. 42, n 1, pp. 104-114.

ELAM A., TERJESEN S; (2010), "Gendered Institutions and Cross-National Patterns of Business Creation for Men and Women”, European Journal of Development Research, n 22, pp. 331-348.

ETTL K., WELTER F. (2010), « Gender, context and entrepreneurial learning », International Journal of Gender and Entrepreneurship, vol. 2, n 2, pp. 108-129.

FERRAZ GOMES A., PIAU SANTANA W.G., PESSOA ARAÚJO U., FONTES MARTINS C.M. (2014), "Empreendedorismo Feminino como Sujeito de Pesquisa", Revista Brasileira de Gestão de Negócios, vol. 16, $\mathrm{n}^{\circ}$ 51, pp. 319-342.

GATEWOOD E.J., BRUSH C.G., CARTER N.M., GREENE P.G., HART M.M. (2009), "Diana: a symbol of women entrepreneurs' hunt for knowledge, money, and the rewards of entrepreneurship", Small Business Economics, vol. 32, pp. 129-144.

GAWEL A. (2013), "Female entrepreneurship in Poland", Poznań University Of Economics Review, vol.13, $\mathrm{n}^{\circ} 1$, pp. 116-130.

GUPTA V.K., TURBAN D.B., WASTI S.A., A. SIKDAR (2009), “The role of gender stereotypes in perceptions of entrepreneurs and intentions to become an entrepreneur" Entrepreneurship Theory and Practice, vol. 33, n², pp. 397-417. 
GUNDRY L., BEN-YOSEPH M., M. POSIG (2002), “Contemporary Perspectives on Women's Entrepreneurship: A Review and Strategic Recommendations”, Journal of Enterprising Culture, vol. $10, \mathrm{n}^{\circ} 1$, pp. 67-86.

HAKIM C. (2000), Work-Lifestyle Choices in the 21st Century. Oxford University Press.

HERNANDEZ E.M. (2006), Extension du domaine de l'entrepreneur... et limites. La Revue des Sciences de Gestion, n $^{\circ} 219$, pp. 17-26.

HILL F.M., LEITCH C.M., HARRISON R.T. (2006), "Desperately Seeking Finance? The Demand for Finance by Women-Owned and -Led Businesses", Venture Capital, vol. 8, n 2, pp. $159-182$.

HISRICH R.D., BRUSH C.G. (1984), "The Woman Entrepreneur: Management Skills and Business Problems", Journal of Small Business Management, January, pp. 30-37.

HISRICH R.D., BRUSH C. G. (1985), The woman entrepreneur: Characteristics and prescriptions for success, Lexington Books.

HISRICH R.D (1986), The Woman Entrepreneur: Characteristics, Skills, Problems, and Prescriptions for Success. In Sexton D. and Smilor,R, ed, The Art and Science of Entrepreneurship, Cambridge: Ballinger.

HOLMEN M., MIN .T.T (2011), "Female Entrepreneurship in Afghanistan”, Journal of Developmental Entrepreneurship, vol. 16, $\mathrm{n}^{\circ} 3$, pp. 307-331.

HOLMQUIST C. (1997), "The other side of the coin or another coin? Women's entrepreneurship as a complement or an alternative?", Entrepreneurship and Regional Development, Vol. 9, pp.179182.

HUGHES K.D. (2003), “Pushed or Pulled? Women's Entry into Self-Employment and Small Business Ownership", Gender, Work and Organization, vol. 10, n 4, pp. 433-454.

HUGHES K.D. (2006), "Exploring Motivation and Success among Canadian Women Entrepreneurs", Journal of Small Business and Entrepreneurship, vol. 19, n 2, pp. 107-120.

HUGHES K., JENNINGS J., BRUSH C., WELTER F. (2012), “Extending Women's Entrepreneurship Research in New Directions", Entrepreneurship Theory and Practice, May, pp. 429-442.

HUGHES K., JENNINGS J. (2012), Global Women's Entrepreneurship Research: Diverse Settings, Questions, and Approaches, Edward Elgar Publishing.

HUMBERT A.L., DREW, E. (2010), “Gender, Entrepreneurship and Motivational Factors in an Irish Context”. International Journal of Gender and Entrepreneurship, vol. 2, n², pp. 173-196.

INTERNATIONAL LABOR ORGANIZATION (ILO) (2004), Global Employment Trends for Women. Geneva.

JEAN M., FORBES C.S. (2012), «An Exploration of the Motivations and Expectation Gap of Mompreneurs », Journal of Business Diversity, vol. 12, n² 2, pp. 112-130.

JENNINGS J., BRUSH C. (2013), "Research on Women Entrepreneurs, Challenges to (and from) the Broader Entrepreneurship Literature?", The Academy of Management Annals, pp. 665-713.

KELlEY D., BRUSH C., GREENE P., HERRINGTON M., ALI A., KEW P. (2015), Women's entrepreneurship, Special Report Global Entrepreneurship Monitor,

KIRKWOOD J. (2009), "Motivational factors in a push-pull theory of entrepreneurship", Gender in Management: An International Journal, vol. 24, n 5, pp. 346-364.

KONRAD A., LANGTON N. (1991), «Sex Differences in Job Preferences, Workplace Segregation, and Compensating Earning Differentials: The Case of Stanford MBAs", Academy of Management Best Papers Proceedings, pp. 368-372.

KORSGAARD S. (2007), «Mompreneurship as a challenge to the growth ideology of entrepreneurship », Kontur, vol. 16, n 1, pp. 42-45.

LANGOWITZ N., MINNITI M. (2007), «The Entrepreneurial Propensity of Women”, Entrepreneurship Theory and Practice, vol. 31, n 3, pp. 341-364.

LEGER-JARNIOU, C., NELSON T. ET CHASSERIO S. (2015), «Perspectives francophones sur les femmes entrepreneures: au-delà des approches comparatives, vers une approche compréhensives », Revue de l'Entrepreneuriat, vol.14, n²-3, pp. 19-30.

LE LOARNE-LEMAIRE, CUPILLARD V., RAHMOUNI BENHIDA B., NIKINA A., SHELTON L.M. (2012), Femme et entrepreneur, c'est possible !, Pearson

LEBEGUE T., (2015), La réussite de carrière entrepreneuriale des femmes, Revue de l'Entrepreneuriat, vol. $14, \mathrm{n}^{\circ} 1$, pp. 93-127.

LEWIS P. (2006), "The Quest for Invisibility: Female Entrepreneurs and the Masculine Norm of Entrepreneurship », Gender, Work and Organization, vol. 13, n 5, pp. 453-469.

LEWIS P. (2013), "The Search for an Authentic Entrepreneurial Identity: Difference and Professionalism among Women Business Owners”, Gender, Work and Organization, vol. 20, n 3, pp. 252-266. 
MARLOW S., CARTER S. (2004), “Accounting for change: professional status, gender disadvantage and self-employment”, Women in Management Review, vol. 19, n¹, pp. 5-17.

MASLOW A. (1954), Motivation and Personality, Harper \& Row Publishers Inc.

MC GOWAN P., LEWIS REDEKER C., COOPER S., GREENAN K. (2012), "Female entrepreneurship and the management of business and domestic roles: Motivations, expectations and realities", Entrepreneurship \& Regional Development, vol. 24, $\mathrm{n}^{\circ}$ 1-2, pp. 53-72.

MINNITI M., ALLEN E., LANGOWITZ N. (2006), The 2005 Global Entrepreneurship Monitor Special Topic Report: Women in Entrepreneurship, Center for Women Leadership, Babson College.

MINNITI M., NAUDE W. (2010), "What Do We Know about the Patterns and Determinants of Female Entrepreneurship across Countries?", European Journal of Development Research, vol. 22, pp. 277-293.

MORRIS B., COXETER R.M. (1995), «Executive Women Confront Midlife Crisis”, Fortune, n 132 , Edition 6.

MORRIS M.H., MIYASAKI N.N., WATTERS C.E., COOMBES S.M. (2006), "The Dilemma of Growth: Understanding Venture Size Choices of Women Entrepreneurs", Journal of Small Business Management, vol. 44, $\mathrm{n}^{\circ}$ 2, pp. 221-244.

MOULT S., ANDERSON A. (2005), «Enterprising Women: Gender and Maturity in New Venture Creation and Development", Journal of Enterprising Culture, vol. 13, n ${ }^{\circ} 3$, pp. 255-271.

NEEGAARD H., SHAW E., CARTER S. (2005), "The impact of gender, social capital and networks on business ownership: a research agenda", International Journal of Entrepreneurial Behavior \& Research, vol. $11, \mathrm{n}^{\circ} 5$, pp. 338 - 357

NEIDER L., (1987) “A preliminary investigation of female entrepreneurs in Florida”, Journal of Small Business Management, vol. 25, $\mathrm{n}^{\circ}$ 3, pp. 22-28.

NEL P., MARITZ A., THONGPROVATI O. (2010), « Motherhood and Entrepreneurship: The Mumpreneur Phenomenon », The International Journal of Organizational Innovation, vol. 3, $\mathrm{n}^{\circ} 1$, pp. 6-34.

ORGANISATION DE COOPÉRATION ET DE DÉVELOPPEMENT ÉCONOMIQUES (OCDE), (2012), Gender equality in education, employment and entrepreneurship: Final report to the MCM 2012. (http://www.oecd.org/social/familiesandchildren/50423364.pdf).

ORHAN M., SCOTT D. (2001), "Why women enter into entrepreneurship: An explanatory model", Women in Management Review, vol.16, n5, p.232-243.

PINES A.M., LERNER M., SCHWARTZ D. (2010), "Gender differences in entrepreneurship, Equality, diversity and inclusion in times of global crisis", Equality, Diversity and Inclusion: An International Journal, Vol.29, n², 186-198.

RICHOMME-HUET, K., VIAL, V., d'ANDRIA, A. (2013). Mumpreneurship: A new concept for an old phenomenon? International Journal of Entrepreneurship and Small Business, Vol.19, n², pp. 251275.

RONSEN M. (2014), "Children and family: A barrier or an incentive to female self-employment in Norway?", International Labour Review, vol. 153, n 2, pp. 337-349.

SCOTT C. (1986), "Why more women are becoming entrepreneurs", Journal of Small Business Management, October, pp. 37-44.

SHAPERO A. (1975), “The Displaced Uncomfortable Entrepreneur”, Psychology Today, n 9 (Nov.), pp. 83-88.

SHAPERO A., SOKOL L. (1982), “The Social Dimensions of Entrepreneurship”, in Kent, C. ; Sexton, D. ; and Vesper, K. (Ed.), Encyclopedia of Entrepreneurship, Prentice-Hall, Englewood Cliffs, New Jersey, pp. 72-90.

SHELTON L. (2006), "Female Entrepreneurs, Work-Family Conflict, and Venture Performance: New Insights into the Work-Family Interface”, Journal of Small Business Management, vol. 44, n², pp. 285-297.

SIMONIN M. (2006), "Women entrepreneurship”, Business Strategy and International Business, Helsinki University of Technology, pp. 91-167.

SNYDER M. (2003), "Bridging the research gap: a profile of women entrepreneurs in Uganda", International Trade Forum Magazine, $\mathrm{n}^{\circ}$ 4. http://www.tradeforum.org/Bridging-the-ResearchGap-A-Profile-of-Women-Entrepreneurs-in-Uganda/

STEVENSON L. (1990), "Some Methodological Problems Associated with Researching Women Entrepreneurs", Journal of Business Ethics; Apr/May, vol. 9, n 4,5, pp. 439-446. 
TORRI M.C., MARTINEZ A. (2014), “Women's empowerment and micro-entrepreneurship in India: Constructing a new development paradigm?”, Progress in Development Studies, vol. 14, n 1, pp. 31-48.

TZEMAH M. (2000). A survey on women characteristics in business. Israel: SMEs Authority cité par ASCHER J. (2012), "Female Entrepreneurship - An Appropriate Response to Gender Discrimination”, Journal of Entrepreneurship, Management and Innovation (JEMI), vol. 8, n 4, pp. 97-114.

VERDUIJN K., ESSERS C. (2013), “Questioning dominant entrepreneurship assumptions: the case of female ethnic minority entrepreneurs", Entrepreneurship \& Regional Development, vol. 25, $\mathrm{n}^{\circ} 7-$ 8, pp. 612-630.

VERHEUL I., VAN STEL A., THURIK R. (2006), "Explaining female and male entrepreneurship at the country level", Entrepreneurship \& Regional Development, vol. 18, pp. 151-183.

VERSTRAETE T., SAPORTA B. (2006). Création d'entreprise et entrepreneuriat. Les Editions de l'ADREG.

VESPER K.H. (1980), New Ventures Strategies, Prentice-Hall.

WEBER M., [1904-1917] Essais sur la théorie de la science, Paris, Pocket, 1992, 181 p.

WELSH D.H., MEMILI E., KACIAK E., AHMED S. (2013), "Sudanese Women Entrepreneurs", Journal of Developmental Entrepreneurship, vol. 18, n 2, pp. 1-18.

WELTER F.F. (2011), "Contextualizing Entrepreneurship-Conceptual Challenges and Ways Forward", Entrepreneurship Theory and Practice, vol. 35, pp. 165-184.

WILLIAMS N., WILLIAMS C. (2012), "Evaluating the socio-spatial contingency of entrepreneurial motivations: A case study of English deprived urban neighbourhoods", Entrepreneurship \& Regional Development, vol. 24, $\mathrm{n}^{\circ}$ 7-8, pp. 661-684.

WINN J. (2004), "Entrepreneurship: Not an easy path to top management for women", Women in Management Review, vol. 19, n 3, pp. 143-153.

ZAHRA S.A (2007), "Contextualizing theory building in entrepreneurship research", Journal of Business Venturing, vol. 22, n³, pp. 443-452. 
Annexe 1 - Eléments de la base de la revue de littérature

\begin{tabular}{|c|c|c|}
\hline 1. $\quad$ Aldrich, Cliff (2003) & 31. Gatewood et al. (2009) & 61. Minniti et al. (2006) \\
\hline 2. Anderson et al. (2010) & 32. Gawel (2013) & 62. Minniti, Naude (2010) \\
\hline 3. Ascher (2012) & 33. Gundry et al. (2002) & 63. Morris et al. (2006) \\
\hline 4. Birley (1989) & 34. Gupta et al. (2009) & 64. Morris, Coxeter (1995) \\
\hline 5. Bowen, Hisrich (1986) & 35. Hakim (2000) & 65. Moult, Anderson (2005) \\
\hline 6. de Bruin et al. (2006) & 36. Hill et al. (2006) & 66. Neider (1987) \\
\hline 7. de Bruin et al. (2007) & 37. Hisrich, Brush (1984) & 67. Nel et al. (2010) \\
\hline 8. Bruni, Gherardi, Poggio (2004) & 38. Hisrich, Brush (1985) & 68. Orhan, Scott (2001) \\
\hline 9. Brush (1990) & 39. Hisrich (1986) & 69. Richomme-Huet, Vial, d'Andria (2013) \\
\hline 10. Brush (1992) & 40. Holmen et al. (2011) & 70. Ronsen (2014) \\
\hline 11. Brush et al. (1999) & 41. Holmquist (1997) & 71. Scott (1986) \\
\hline 12. Brush et al. (2001) & 42. Hughes (2003) & 72. Shelton (2006) \\
\hline 13. Brush, Cooper (2012) & 43. Hughes (2006) & 73. Simonin (2006) \\
\hline 14. Brush, de Bruin, Welter (2009) & 44. Hughes et al. (2012) & 74. Snyder (2003) \\
\hline 15. Buttner, Moore (1997) & 45. Hughes, Jennings (2012) & 75. Stevenson (1990) \\
\hline 16. Carter (2000) & 46. Humbert, Drew (2010) & 76. Torri, Martinez (2014) \\
\hline 17. Cobe, Parlapiano (2002) & 47. Jean, Forbes (2012) & 77. Tzemah (2000) \\
\hline 18. Cornet, Constantinidis (2004) & 48. Jennings, Brush (2013) & 78. Verduijn, Essers (2013) \\
\hline 19. Coughlin, Thomas (2002) & 49. Kelley et al. (2015) & 79. Verheul et al. (2006) \\
\hline 20. Cromie (1987) & 50. Kirkwood (2009) & 80. Welsh et al. (2013) \\
\hline 21. Cromie, Hayes (1988) & 51. Konrad, Langton (1991) & 81. Williams, Williams (2012) \\
\hline 22. d'Andria (2014) & 52. Korsgaard (2007) & 82. Winn (2004) \\
\hline 23. Davis, Abdiyeva (2012) & 53. Langowitz, Minniti (2007) & \\
\hline 24. Duberley, Carrigan (2012) & 54. Léger-Jarniou, Nelson, Chasserio (2015) & \\
\hline 25. Ducheneaut (1997) & 55. Le Loarne-Lemaire et al. (2012) & \\
\hline 26. Eijdenberg, Masurel (2013) & 56. Lebègue (2015) & \\
\hline 27. Ekinsmyth (2011) & 57. Lewis (2006) & \\
\hline 28. Elam, Terjesem (2010) & 58. Lewis (2013) & \\
\hline 29. Ettl, Welter (2010) & 59. Marlow, Carter (2004) & \\
\hline 30. Ferraz Gomes et al. (2014) & 60. Mc Gowan et al. (2012) & \\
\hline
\end{tabular}

